\title{
Interactive comment on "On surface fluxes at night - the virtual chamber approach" by Bruce B. Hicks et al.
}

\section{Bruce B. Hicks et al.}

nebila.lichiheb@noaa.gov

Received and published: 17 April 2020

We thank Dr. Thomas Foken for his valuable comments. Please see below our answers to the reviewers. Various ways of improvement of the manuscript will be suggested based on these answers in order to adjust the manuscript in the way it was envisioned.

Dr. Thomas Foken points out that the analysis might benefit from additional attention to the stability regime. In accordance with this, the analysis has been repeated, with the conclusion that the amended Zimbabwe results do indeed appear more consistent. While doing this, the Ohio data were also examined from the same perspective, with no major changes resulting. However, the opportunity has been taken to modify both Figures 4 and 7 so that they do indeed represent the different datasets in the same 
way. Previously Fig. 4(b) plotted a subset of the data for which confidence was highest, whereas Fig. 7(b) plotted all results. Now, all results are plotted in both diagrams. The results with highest confidence are summarized in the text.

\section{Response to the Reviewer \#2:}

The authors present an interesting approach of using a "virtual chamber" to discuss the influence of the complicated condition of the atmospheric turbulence at night on chamber measurements. This is an important contribution to making chamber measurements more representative, even if some problems are still open like the validation. Furthermore, the design of experiments for virtual chambers must be updated. The three experiments used can only provide a first guess. A discussion in the community would be helpful; perhaps we have better-equipped experiments for further studies. For the validation I propose eddy-covariance measurements with high resolution in time on the basis of a wavelet analysis (Schaller et al. 2019). The nature of the problem is such that we would be most happy with an independent test. However, we are pursuing the matter ourselves, without yet discovering new datasets. It is our interpretation that the complexity of the circumstances in which our virtual chamber approach would work best is such that most mainstream meteorologists would take all possible steps to avoid it.

The authors highlighted problems like low level jets and breaking gravity waves that affect the turbulent exchange significantly, mainly after midnight. Closed chambers cannot usually measure (or only partly measure) these higher exchange rates. In the discussion I am missing another effect: at night, closed chambers have a longwave net radiation near zero. That means they always have neutral stratification, whereas outside a strong stable stratification exists (Riederer et al. 2014). Please discuss this effect too. In Figure $7 \mathrm{~b}$, before midnight such a radiation effect for the closed chamber may be possible. However, the virtual chamber cannot reproduce the radiation effect and I am probably seeing the influence of a strong stable stratification that is very typical before midnight, while after midnight it is often the case that condensation (dew)

Printer-friendly version

Discussion paper 
reduces the degree of stability.

Certainly, the mixing regime inside a chamber will differ greatly from the outside air. This will be the case whether or not the air in the chamber is mechanically stirred. We want to avoid discussing this in detail, but the identification of the issue and its relevance to Figure 7 (b) caused us to examine the data sets and their analysis again. The new scrutiny revealed instances of not-credible measures, constituting unexplained extremes in the data sequences. Hence, we chose to impose a data exclusion criterion. This entailed computing the averages and standard deviation of sequential packets of observations. If any particular data point differed from its preceding average by more than four standard deviations, it has now been excluded. This criterion has been applied to all of the sequences of data used. It had no consequences on the Ohio case, but resulted in the elimination of several data outliers in the Zimbabwe case.

The revised Fig 7 reflects the application of this criterion

We have also updated the following text in the manuscript

As before, there is no convincing reason to prefer the y-type results over the $x$-type, even though the near-zero results (x-type) indicated in the diagram are disturbing. If $x$-type and $y$-type results are averaged (as was the case in consideration of the Ohio dataset), the resulting estimate of the $\mathrm{CO} 2$ efflux for the Zimbabwe November data is $20.7 \pm 4.8 \mu \mathrm{g} \mathrm{m}-2 \mathrm{~s}-1$ for the area sown with maize, and $24.3 \pm 2.5 \mu \mathrm{g} \mathrm{m}-2 \mathrm{~s}-1$ for the fallow. At the time of these measurements, the maize had not yet fully emerged and the fallow field was poorly vegetated (with weeds). Concentration and virtual temperature data refer (as before) to a height of about $1 \mathrm{~m}$ above ground level.

Some minor remarks: 1. Line 33: Recently eddy-covariance is used instead of eddycorrelation.

We have replaced the term "eddy-correlation" by the term "eddy-covariance" in the whole manuscript. 2. Line 109: I think sigma w is an important parameter to describe

Printer-friendly version

Discussion paper
Interactive comment 
the influence of turbulence on fluxes. Because the basic instrumentation of the three experiments is a Bowen-ratio installation, please make some remarks about the measurements of sigmaw.

Indeed, we have added the following text to the manuscript:

In practice, the wind speed $u$ is an output of the sonic anemometer, as is the standard deviation of the vertical wind component $\sigma \mathrm{w}$. The rate of change of concentration, $\mathrm{dC} / \mathrm{dt}$, is conveniently computed from the initial time sequence of measurement as:

$\mathrm{dC} / \mathrm{dt}=(\mathrm{Cn}+1-\mathrm{Cn}-1) /(\mathrm{tn}+1-\mathrm{tn}-1)(4)$ where the measurement level of $\mathrm{C}(\mathrm{zc})$ is such that the lack of turbulence indicated by the sonic anemometer will also be indicative of a lack turbulent exchange at the height of measurement of $\mathrm{C}$.

We have also added the following text to the manuscript:

The discussion above relates to a situation in which $\sigma \mathrm{w}$ data are routinely available, synchronized with the $\mathrm{C}$ measurements. Such data are regularly provided by modern three-dimensional sonic anemometers, whose deployment is usually associated with the determination of fluxes directly, by covariance between concentrations and the vertical wind speed component. In the present case, the requirement that deployment must follow guidelines for accurate flux determination can be relaxed, because it is only the magnitude of $\sigma \mathrm{w}$ that is needed. In Fig. 1 (b) the measurements are assumed to be at a height (za) above that of C-measurement (zc). It is assumed that as $\sigma \mathrm{w}$ trends to zero at height za, so it does at a lower height za, or $h$. In the lack of measurements of $\sigma \mathrm{W}$, virtual temperature gradients or TKE could be used equivalently.

3. Line 144: The linear gradient should be the first guess.

We agree that wind-tunnel (and other) experience suggests that the linear gradient is Printer-friendly version the better approximation. 4. Line 145: Please explain the square root in connection with Figure 1 more clearly.

Discussion paper The matter has been explained more carefully in the description of Figure 1. 
5. Fig. 4 and 7: Because I am color-blind, I see nothing (only with scientific background and context am I able to form an impression). Could you please use black and grey instead of red and green?

As previously explained, the analysis has been repeated and Figures 4 and 7 has been corrected and clarified in the revised manuscript by using black and grey curves with solid and open circles.

We have also updated the following text in the manuscript

Consideration of only the most robust results (those for which $R 1.232>0.25$ ) yield an average soil efflux rate of $2.55 \pm 0.31 \mu \mathrm{g} \mathrm{m}-2 \mathrm{~s}-1$ for the previously tilled surface, and $2.25 \pm 0.32 \mu \mathrm{g} \mathrm{m}-2 \mathrm{~s}-1$ for the untilled. The most likely averages of CO2 nocturnal emissions from Ohio agricultural soils are therefore indicated to be about $2.5 \mu \mathrm{g} \mathrm{m}-2$ $\mathrm{s}-1$ for the conditions of the current test (November, after harvest), regardless of whether the surface was previously tilled.

Please also note the supplement to this comment: https://www.biogeosciences-discuss.net/bg-2019-393/bg-2019-393-AC2-

supplement.pdf

Interactive comment on Biogeosciences Discuss., https://doi.org/10.5194/bg-2019-393, 2019. 


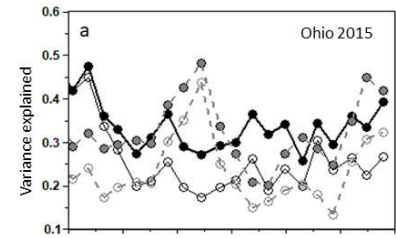

Interactive

comment

Figure 4. (a) The average variation during the night of the proportion of variance in $d C / d t$ ('closed chamber'; solid circles) and ( $d C / d t)^{2}$ ('open chamber'; open circles) that can be accounted for by the present statistical treatment. Black symbols relate to a previously-tilled area, grey to an adjacent area not tilled. (b) the estimates of surface efflux rates derived from the same analysis.

Fig. 1. 


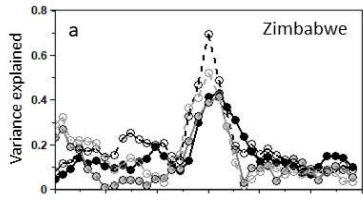

Interactive

comment

Figure 7. As in Figure 4, with black points relating to a fallow field and grey to an adjoining area recently planted with maize (on 8 November, 2013). The analysis procedure is identical with that leading to Figure 4. The period represented here is the first two weeks of the month of November. Solid points indicate results obtained using the closed-chamber approximation described here, open points represent vented-chamber approximations.

Fig. 2. 